\title{
An Investigation Into the Three-Dimensional Stress-Birefringence-Strain Relationship in Elastomers
}

\author{
ELLEN M. ARRUDA and PHILLIP A. PRZYBYLO \\ The Department of Mechanical Engineering and Applied Mechanics \\ The University of Michigan \\ Ann Arbor, Michigan 48109-2125
}

\begin{abstract}
The statistical theory of the birefringence of an individual non-Gaussian elastomer chain is used together with a chain network description of rubber elasticity to develop a relationship among the strain, birefringence, and stress in elastomers, valid for large deformations under generalized strain states. The result is a fully three-dimensional internal variable based constitutive model of rubber elasticity in which measurement of the elastomeric birefringence during straining in one deformation state characterizes the optically anisotropic response of the elastomer. Simultaneous measurement of the stress vs. strain response provides the rubbery modulus and limiting network extensibility properties needed to completely characterize the mechanical anisotropy of the material. Once characterized using the single, large deformation experiment, the birefringence and stress responses of the elastomer in other deformation states may then be predicted without adjusting any model parameters. The theory is compared to experimental studies from the literature of large strain deformations of elastomers in uniaxial tension and compression for which the exhibited birefringence and stress responses of deforming elastomers have been simultaneously recorded.
\end{abstract}

\section{INTRODUCTION}

$\mathrm{C}$ onstitutive models of the large strain, nonlinear elastic behavior of rubbery polymers have been in existence for over fifty years; early models included the phenomenological invariant-based approaches such as that developed by Mooney (1) and the statistical mechanics models by investigators such as Flory and Rehner (2), Flory and Erman (3), Ullman (4), Erman (5), Stepto (6), and Arruda and Boyce (7). Concurrently, statistical mechanics based birefringence vs. stretch theories have been developed based on the same principle as the rubber elasticity models: that the orientation of molecular chains during stretching results in a measurable response from the anisotropic elastomer (8-11). In the case of the birefringence theories, the response is due to the anisotropic polarizability of the stretched network for which incident light is retarded by unequal amounts in various directions as it passes through the material. These two statistical formulations, rubber elasticity and the theory of elastomer birefringence, have remained as separate descriptors of the elastomeric response to imposed stretch states. The theories of rubber birefringence and rubber elasticity have previously been combined only for the case of small strains
(Gaussian chains) yielding the familiar stress-optic law

$$
\Delta \eta_{1-2}=\left(\frac{2 \pi \alpha}{45 k \Theta} \frac{\left(\eta_{0}^{2}+2+2\right)^{2}}{\eta_{0}}\right)\left(\sigma_{1}-\sigma_{2}\right)
$$

where $\Delta \eta_{1-2}$ is the birefringence (the difference in the amount of retardation of light in orthogonal directions one and two $)\left(\sigma_{1}-\sigma_{2}\right)$ is the stress difference in the 1-2 plane and the term $\left(2 \pi \alpha / 45 \mathrm{k} \Theta\left(\eta_{0}^{2}+\right.\right.$ $2)^{2} / \eta_{0}$ ) is $C$, the stress-optic coefficient, which is constant according to theory using Gaussian chains and affine junction point motion. In $C, \alpha$ is the polarizability anisotropy of the statistical structural unit or rigid link, $\eta_{0}$ is the mean refractive index, $k$ is Boltzmann's constant and $\Theta$ is absolute temperature.

As the Treloar data (12) in Fig. 1 show, the birefringence vs. stress response deviates from linearity at moderate strains. Previous investigations have led to nonlinear stress optic laws $(9,12-16)$, but for various reasons these existing theories are not adequate as three-dimensional constitutive models of either rubber elasticity or rubber birefringence. Models such as that of Flory and Rehner (2) or the Kuhn and Grün (13) model later investigated by Treloar (12) have 


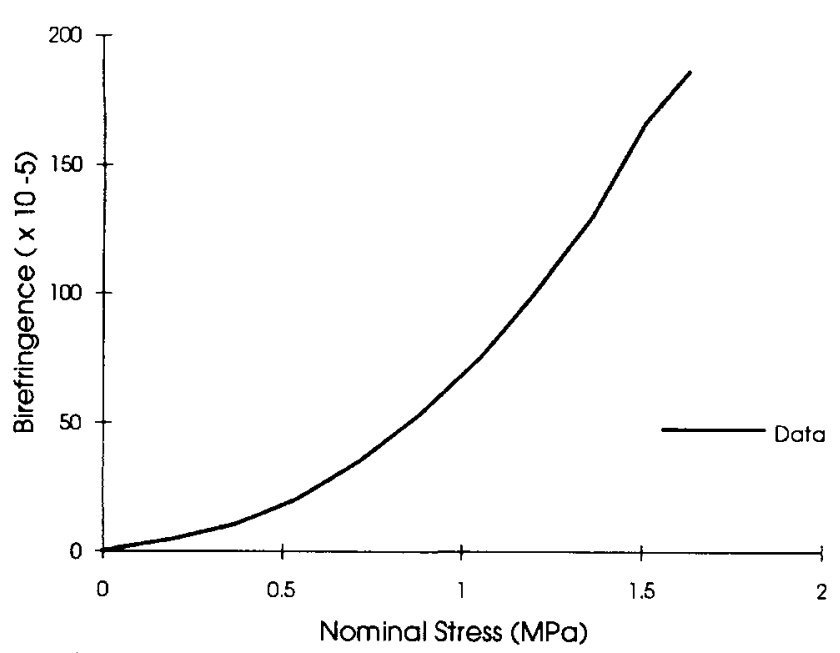

Fig. 1. Treloar data (12) in uniaxial tension plotted as birefringence us. nominal stress showing the non-linearity of the stress-optic law at intermediate stretches.

previously been shown by Arruda and Boyce (7) to not be predictive of the deformation state dependent response of elastomers at intermediate and large strains. Similarly, the Flory and Erman (3) model requires adjustment of at least one of its parameters in order to capture both uniaxial tension and uniaxial compression data. The models based on distributions of chains $(13,14,16)$ are mathematically very cumbersome to use as three-dimensional constitutive models; to the authors' knowledge these models have not been compared to data in various deformation states and it is unknown whether any of these models would correctly capture the response in a given deformation state without adjusting material properties.

The current modeling approach uses the only existing network constitutive model of rubber deformation that has been successfully compared with data from several elastomers at large strains and in various strain states (7) to predict without adjusting parameters the mechanical anisotropy seen in actual experimental results. The model is virtually indistinguishable from other well-known models at small stretches $(2,13)$. The optical anisotropy of the constitutive network description of Arruda and Boyce (7) is considered in the present study to produce an internal variable-based model of elastomeric deformation, valid for generalized strain states.

\section{MODELING}

\section{The Birefringence of an Elastomeric Network}

The birefringence is related to the network polarizability anisotropy following the work of Kuhn and Grün (13)

$$
\Delta \eta_{1-2}=\eta_{1}-\eta_{2}=\frac{2 \theta}{9 \eta_{0}}\left(\eta_{0}^{2}+2\right)^{2}\left(\beta_{1}-\beta_{2}\right)
$$

where $\Delta \eta_{1-2}$ is the birefringence or difference in refractive indices in the $1-2$ plane, $\eta_{0}=\frac{1}{3}\left(\eta_{1}+\eta_{2}+\eta_{3}\right)$ is the mean refractive index of the medium and $\left(\beta_{1}-\right.$ $\beta_{2}$ ) is the network polarizability anisotropy in the $1-2$ plane.

Similar expressions may be written for the $1-3$ and 2-3 planes:

$$
\begin{aligned}
& \Delta \eta_{1-3}=\eta_{1}-\eta_{3}=\frac{2 \pi}{9 \eta_{0}}\left(\eta_{0}^{2}+2\right)^{2}\left(\beta_{1}-\beta_{3}\right) \\
& \Delta \eta_{2-3}=\eta_{2}-\eta_{3}=\frac{2 \pi}{9 \eta_{0}}\left(\eta_{0}^{2}+2\right)^{2}\left(\beta_{2}-\beta_{3}\right)
\end{aligned}
$$

Together, Eqs 2, 3, and 4 completely describe the optical anisotropy of the medium for generalized stretch states.

The network polarizability anisotropy is to be formulated from the network structure using non-Gaussian statistical descriptions of the polarizability anisotropy of network chains in a network geometry. Previous descriptions of network polarizability anisotropy include those of Kuhn and Grün (13), who used a total assembly of non-Gaussian chains model that required integration over all chain orientations in space. Their analysis relied on the simplifications afforded by considering uniaxial tension deformation (i.e., axisymmetry) and by approximating the nonGaussian chain statistics, and is not useful for general strain states nor valid at large stretches. Treloar (12) invoked a three-chain model that has previously been shown to not be accurately predictive of the constitutive response of rubber materials in various strain states (7). Smith and Puett (15) examined the region just beyond the limits of Gaussian statistics and integrated over a distribution of chains that differed from the total assembly of chains distributions. Treloar and Riding (14) later examined the total assembly of chains model for biaxial strain states and large strains. Because of the mathematical complexity of their model, Treloar and Riding used a threeterm approximation to the non-Gaussian chain, adversely affecting large deformation results. In the current study, a model for elastomer birefringence is developed using an eight-chain formulation (7) to account for the contributions of chain polarizability anisotropy from individual non-Gaussian chains to the entire network polarizability anisotropy. This approach offers the advantage that the network used provides an accurate constitutive description of rubber elastic deformation for generalized strain states over the entire extensibility range of elastomers (7).

Following the traditional statistical mechanics of rubber elasticity (10), an individual elastomer chain is modeled as a number, $N$, of rigid links of length, $l$, between chemical crosslinks. These links are joined together assuming a statistical distribution of link angles as in Langevin statistics. Each link is assumed to be optically transversely anisotropic; the anisotropy 
is defined by the link polarizability along is axis, $\alpha_{\|}$, and that perpendicular to its axis, $\alpha_{\perp}$. The polarizability anisotropy of a single chain made up of $N$ of these rigid links is found to be (13)

$$
\gamma_{\text {chain }}=\gamma_{\|}-\gamma_{\perp}=N\left(\alpha_{\|}-\alpha_{\perp}\right)\left\{1-\frac{3 \frac{r_{\text {chain }}}{N l}}{\mathscr{L}^{-1}\left(\frac{r_{\text {chain }}}{N l}\right)}\right\}
$$

where $\gamma_{\text {chain }}=\gamma_{\|}-\gamma_{\perp}$ is the chain polarizability anisotropy measured as the difference between polarizabilities along the chain axis and normal to the chain axis, $r_{c h a i n}$ is the chain vector length and the term $\mathscr{L}^{-1}\left(r_{\text {chain }} / N\right)$ is the inverse Langevin function defined by

$$
\mathscr{L}(\chi)=\operatorname{coth} \chi-\frac{1}{\chi}
$$

The single chain having a polarizability anisotropy given by $E q 5$ is incorporated into the network shown in Fig. 2 (7). The chains in the network have equal magnitudes, $r_{\text {chain }}$, and undergo equal stretches, $\lambda_{\text {chain }}$, upon imposing a principal stretch state $\lambda_{1}$, $\lambda_{2}, \lambda_{3}$ to the elastomeric network. The model assumes incompressibility, requiring that the applied stretches satisfy the constraint

$$
\lambda_{1} \lambda_{2} \lambda_{3}=1
$$

The chain length under any deformation state $\lambda_{1}$, $\lambda_{2}, \lambda_{3}$ is

$$
r_{\text {chain }}=\frac{\sqrt{N} l}{\sqrt{3}} I_{1}^{1 / 2}
$$

where $I_{1}=\lambda_{1}^{2}+\lambda_{2}^{2}+\lambda_{3}^{2}$, is the first principal stretch invariant. The unstretched length of all chains in the
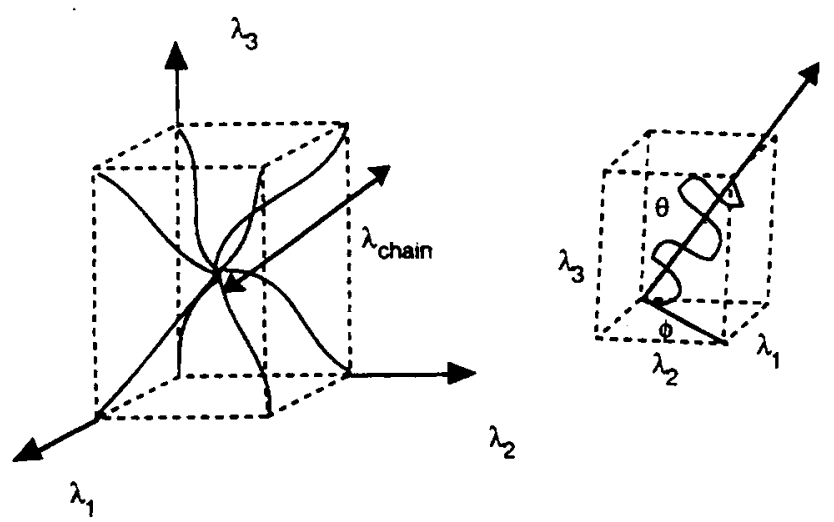

Fig. 2. Schematic illustration of the eight-chain network (7) used here to describe the optical anisotropy of an elastomer network, and hence, its birefringence, in addition to describing the mechanical anisotropy that arises from stretching the network by $\lambda_{1}, \lambda_{2}$ and $\lambda_{3}$. model is given by random walk statistics as $r_{0}=\sqrt{N} l$; thus the chain stretch is given by

$$
\lambda_{\text {chain }}=\frac{1}{\sqrt{3}} I_{1}^{1 / 2}
$$

Letting $\alpha=\alpha_{\|}-\alpha_{\perp}$ denote the link anisotropy and substituting chain stretch in place of chain length, Eq 5 becomes

$$
\gamma_{\text {chain }}=N \alpha\left\{1-\frac{3 \frac{\lambda_{\text {chain }}}{\sqrt{N}}}{\mathscr{L}^{1}\left(\frac{\lambda_{\text {chain }}}{\sqrt{N}}\right)}\right\}
$$

This chain is incorporated into the network geometry depicted in Fig. 2, the orientation of the chains in the network may be described by the angles $\theta$ and $\phi$ in Fig. 2. These angles are related to the applied stretches, $\lambda_{1}, \lambda_{2}, \lambda_{3}$, as follows

$$
\begin{aligned}
& \cos ^{2} \theta=\frac{\lambda_{1}^{2}}{\lambda_{1}^{2}+\lambda_{2}^{2}+\lambda_{3}^{2}} \\
& \sin ^{2} \theta=\frac{\lambda_{2}^{2}+\lambda_{3}^{2}}{\lambda_{1}^{2}+\lambda_{2}^{2}+\lambda_{3}^{2}} \\
& \cos ^{2} \phi=\frac{\lambda_{2}^{2}}{\lambda_{2}^{2}+\lambda_{3}^{2}} \\
& \sin ^{2} \phi=\frac{\lambda_{3}^{2}}{\lambda_{2}^{2}+\lambda_{3}^{2}}
\end{aligned}
$$

The polarizability tensor components along the principal stretch directions for the chain described by angles $\theta$ and $\phi$ are as follows (17)

$$
\begin{aligned}
& \beta_{11}=\gamma_{\|} \cos ^{2} \theta+\gamma_{\perp} \sin ^{2} \theta \\
& \beta_{22}=\left(\gamma_{\|}-\gamma_{\perp}\right) \sin ^{2} \theta \cos ^{2} \phi+\gamma_{\perp} \\
& \beta_{33}=\left(\gamma_{\|}-\gamma_{\perp}\right) \sin ^{2} \theta \sin ^{2} \phi+\gamma_{\perp} \\
& \beta_{12}=\left(\gamma_{\|}-\gamma_{\perp}\right) \sin \theta \cos \theta \cos \phi \\
& \beta_{23}=\left(\gamma_{\|}-\gamma_{\perp}\right) \sin ^{2} \theta \sin \phi \cos \phi \\
& \beta_{13}=\left(\gamma_{\|}-\gamma_{\perp}\right) \sin \theta \cos \theta \sin \phi .
\end{aligned}
$$

The contributions of the polarizability anisotropy of all eight chains to the network polarizability tensor are summed to give expressions for the network polarizability anisotropy. In the 1-2 plane the result is the following

$$
\beta_{11}-\beta_{22}=n \alpha N\left(\frac{\lambda_{1}^{2}-\lambda_{2}^{2}}{3 \lambda_{\text {chain }}^{2}}\right)\left\{1-\frac{3 \frac{\lambda_{\text {chain }}}{\sqrt{N}}}{\mathscr{L}^{-1}\left(\frac{\lambda_{\text {chain }}}{\sqrt{N}}\right)}\right\}
$$

where $n$ is the chain density. This summation over the eight-chain geometry also results in $\beta_{12}=\beta_{23}=$ 
$\beta_{13}=0$; therefore the tensor components above are principal values and the left hand side of $E q 13$ may be denoted as $\beta_{1}-\beta_{2}$. Equations 2 and 11 combine to give an expression for the birefringence in the $1-2$ plane,

$$
\begin{aligned}
\Delta \eta_{1-2}= & \frac{2 \pi}{9} \frac{\left(\eta_{0}^{2}+2\right)^{2}}{\eta_{0}} n \alpha N\left(\frac{\lambda_{1}^{2}-\lambda_{2}^{2}}{3 \lambda_{\text {chain }}^{2}}\right) \\
& \cdot\left\{\begin{array}{l}
3 \frac{\lambda_{\text {chain }}}{\sqrt{N}} \\
\left.1-\frac{\mathscr{L}^{1}\left(\frac{\lambda_{\text {chain }}}{\sqrt{N}}\right)}{\sqrt{N}}\right)
\end{array}\right.
\end{aligned}
$$

\section{Birefringence and Rubber Elasticity}

Langevin chain statistics are also employed to obtain an expression for the conformational entropy of a stretched chain modeled as in the proceeding section to contain a number $N$ of statistical rigid links of length $L$ The entropy of the chain is given as

$$
\begin{aligned}
s_{\text {chain }}= & k\left[c-N\left(\frac{\lambda_{\text {chain }}}{\sqrt{N}} \mathscr{L}^{1}\left(\frac{\lambda_{\text {chain }}}{\sqrt{N}}\right)\right.\right. \\
& \left.\left.+\ln \frac{\mathscr{L}^{-1}\left(\frac{\lambda_{\text {chain }}}{\sqrt{N}}\right)}{\sinh \mathscr{L}^{-1}\left(\frac{\lambda_{\text {chain }}}{\sqrt{N}}\right)}\right)\right]
\end{aligned}
$$

where $\lambda_{\text {chain }}$ is the chain stretch defined in Equation $9, k$ is Boltzmann's constant, and $c$ is a constant (10). A force arises upon stretching to resist the entropy change that favors the randomly coiled chains. The force on a chain stretched by an amount $\lambda_{\text {chain }}$ is

$$
f_{\text {chain }}=-\sqrt{N} \Theta\left(\frac{\delta s_{\text {chain }}}{\delta \lambda_{\text {chain }}}\right)
$$

where $\Theta$ is the absolute temperature.

The elasticity of the entire network is found by summing contributions of forces on all chains in the network analogously to the manner in which the total network optical anisotropy was established earlier. The procedure results in expressions for the difference in the in-plane principal stresses as functions of imposed principal stretches (7)

$$
\sigma_{1}-\sigma_{2}=\frac{n k \Theta}{3} \sqrt{N} \mathscr{L}^{-1}\left(\frac{\lambda_{\text {chair }}}{\sqrt{N}}\right) \frac{\lambda_{1}^{2}-\lambda_{2}^{2}}{\lambda_{\text {chain }}}
$$

where all quantities have been described in the previous section.
Equations 12 and 15 may be combined to give the stress-optic law for the current theory

$$
\begin{aligned}
\frac{\Delta \eta_{1-2}}{\sigma_{1}-\sigma_{2}}= & \frac{2 \pi}{9} \frac{\alpha \sqrt{N}}{k \Theta} \frac{\left(\eta_{0}^{2}+2\right)^{2}}{\eta_{0}}\left(\frac{1}{\lambda_{\text {chain }}}\right) \\
& {\left[\frac{\left(\left\{\frac{\lambda_{\text {chain }}}{\sqrt{N}}\right)\right.}{1-\frac{\mathscr{L}^{-1}\left(\frac{\lambda_{\text {chain }}}{\sqrt{N}}\right)}{\mathscr{L}^{-1}\left(\frac{\lambda_{\text {chain }}}{\sqrt{N}}\right)}}\right] }
\end{aligned}
$$

The current formulation of elastomeric birefringence and elasticity predicts that birefringence is not linear with stress at large stretches. The stress-optic law may be rearranged and the constants combined so that the difference in principal stresses may be written as a function of birefringence and stretch

$$
\begin{aligned}
\sigma_{1}-\sigma_{2}= & \Delta \eta_{1-2} \frac{9}{2 \pi} \frac{k \Theta}{\alpha \sqrt{N}} \frac{\eta_{0}}{\left(\eta_{0}^{2}+2\right)^{2}}\left(\lambda_{\text {chain }}\right) \\
& {\left[\frac{\mathscr{L}^{-1}\left(\frac{\lambda_{\text {chain }}}{\sqrt{N}}\right)}{\left\{1-\frac{\lambda_{\text {chain }}}{\sqrt{N}}\right)}\right] }
\end{aligned}
$$

The familiar stress-optic coefficient is recovered if the terms containing the Langevin function are approximated by the first term in a series expansion [see, for example Treloar (10) for the series]. Equation 17 reduces to

$$
\sigma_{1}-\sigma_{2}=\left(\frac{45 k \Theta}{2 \pi \alpha} \frac{\eta_{0}}{\left(\eta_{0}^{2}+2\right)^{2}}\right) \Delta \eta_{1-2}
$$

in the small stretch limit, where the quantity $(2 \pi \alpha /$ $\left.45 k \Theta\left(\eta_{0}^{2}+2\right)^{2} / \eta_{0}\right)$ is the familiar stress-optic coefficient. The current theory predicts that at small deformations stress is linearly proportional to birefringence, in accordance with Gaussian theories of rubber birefringence. Beyond moderate stretch levels the non-Gaussian stress-optic law deviates from linearity in accordance with the data in Fig. 1.

\section{Determination of Material Properties}

Equation 12 for the elastomer birefringence contains all material properties required for determining the stress response in addition to the birefringence response. Four material properties are involved, three of which are defined in terms of properties of the statistical chain. 
Characterization of the birefringence vs. stretch response requires the mean refractive index of a medium, $\eta_{0}$, which may be found in polymer handbooks; see, for example, Van Krevelen (17). The remaining three properties, $n, N$, and $\alpha$, are defined for the statistical chain and chain network used to model the actual polymer molecule and structure. These may be determined rigorously from various descriptors of the polymer molecules if, in actuality, the polymer molecules formed a "model" network-a monodisperse, defect-free structure of known, constant crosslink functionality. Such calculations require the assumption that the actual molecule contains freely rotating bonds; allowances for steric constraints can also be incorporated into the calculations. The real polymer structure varies from the model network quite substantially, resulting in deviations in the statistical material properties from values calculated based on the molecular polymer descriptors. The link polarizability anisotropy, $\alpha$, may be determined by a tensorial summation over all bonds in the link according to the theory of Nagai (18).

$$
\alpha=\frac{3}{2} \sum_{i=1}^{n} \frac{\left\langle\mathbf{r}^{\mathbf{T}} \hat{\mathbf{a}}_{i} \mathbf{r}\right\rangle_{0}}{\left\langle r^{2}\right\rangle_{0}}
$$

where $\mathbf{r}$ is the vector length of group $i, \hat{\mathbf{a}}_{i}$ is the polarizability anisotropy of the group, and $r$ is the magnitude of the group vector length. Summation is performed over all groups comprising the statistical link in the freely jointed chain. Values for the optical anisotropies of several monomer groups are found in Van Krevelen (18).

For an idealized octafunctional structure in which all chains form in the network, the polymer molecule provides $\eta_{0}$ directly and $\alpha$ through the assumption of the existence of an equivalent randomly jointed statistical chain for modeling a freely rotating molecular chain. The product $n N$ is similarly found. Therefore it remains that the actual characterization of an elastomer would require a curve fit of the birefringence vs. stretch data in uniaxial tension via $\mathrm{Eq} 12$ to uniquely determine $n$ and $N$. The stress-stretch response of $E q 17$ is then completely characterized from the fitting of the birefringence data; the same material properties used in $E q 12$ to characterize the material's birefringence versus stretch response are used to predict the stress-stretch response of the model network elastomer.

In reality, the elastomers available for testing have not formed model network structures. They contain defects such as loops and free ends, and the molar mass between crosslinks is not a constant. Also, the crosslink functionality is not constant throughout the material. Because the model structure used (7) is an idealization of a real network, characterization of the material response requires both the birefringence and stress curves in one deformation state such as uniaxial tension in order to determine $n, N$, and $\alpha$. The advantage of this model is that with the material so characterized using the uniaxial tension re- sponses, predictions may be made in other deformation states without changing these material properties. Moreover, the model is valid for deformations beyond the Gaussian range.

\section{COMPARISON WTH EXPERIMENTAL RESULTS}

We have investigated existing data in the literature for which simultaneous measures of birefringence and load were recorded versus stretch. Early experiments of this type were conducted on natural rubber by Treloar (12), in which the birefringence vs. stretch and nominal stress vs. stretch responses were recorded for uniaxial tension. Treloar's results (12) are included in Fig. 3 along with the results of our simulations using $E q 12$, together with the following values of the model material properties:

$$
\begin{aligned}
& \eta_{\mathrm{O}}=1.5205 \\
& \alpha=51.1 \times 10^{-31} \mathrm{~m}^{3} \\
& n=7.25 \times 10^{25} \mathrm{~m}^{-3} \\
& N=25.0
\end{aligned}
$$

to provide a characterization of this material. We illustrate in Fig. 3 the success with which we can capture the nonlinear uniaxial birefringence vs. stretch data using $E q$ 14. The calculated birefrin-
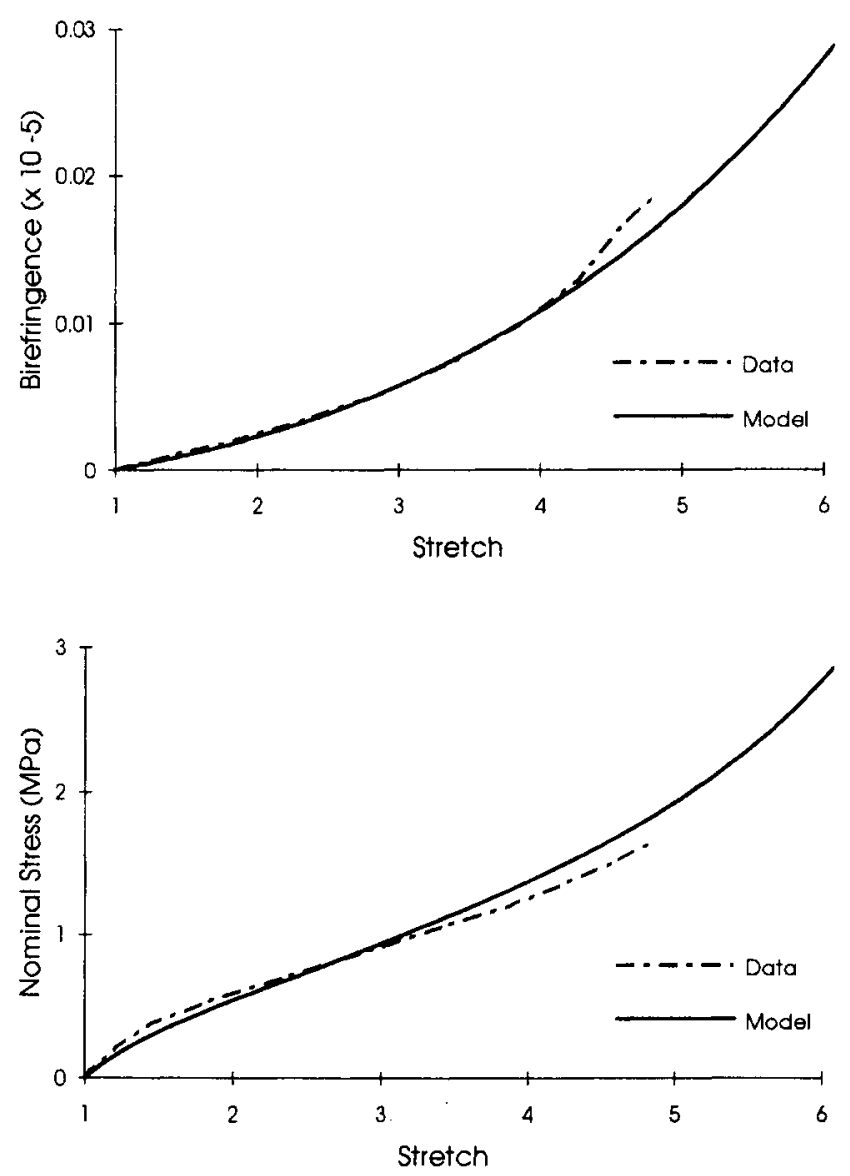

Fig. 3. Birefringence vs. stretch and nominal stress vs. stretch responses of natural rubber in uniaxial tension. Data from Treloar (12). Simulations used Eq 14 for birefringence, and $\mathrm{Eq} 19$ for nominal stress. 
gence values from $\mathrm{Eq} 12$ were then used in $\mathrm{Eq} 17$ to produce the simulated nominal stress vs. stretch curve in Fig. 3. Both the birefringence vs. stretch and nominal stress vs. stretch responses are seen to be well described by the current theory for the entire data ranges in uniaxial tension. ${ }^{1}$ Equation 14 provides an accurate description of the birefringence vs. stretch data up to a stretch of about 4.5 at which point the data and simulation diverge. The calculated birefringence from $E q 12$ (rather than the actual data) are used to predict the stress-stretch response, yielding an accurate fit throughout the stretch range recorded. It is suspected that the birefringence vs. stretch curves diverge at the largest stretches recorded because of inaccurate transcription of the 1947 data to Fig. 3.

The value of $\eta_{0}$ used in Fig. 3 is that given by Treloar (12) for natural rubber. The additional material parameters were chosen to best fit the birefringence and stress curves. We have also examined the data of Flory and Erman $(3,8,9,19)$, who tested two PDMS networks that differed somewhat in mechanical and optical responses because they were synthesized under different conditions. In Fig. 4 are the uniaxial tension nominal stress vs. stretch and birefringence vs. stretch responses for their PDMS(A) material. Included in Fig. 4 are our simulations of these responses using the following values of the model material properties:

$$
\begin{aligned}
& \eta_{0}=1.4074 \\
& \alpha=5.4 \times 10^{-31} \mathrm{~m}^{3} \\
& n=4.55 \times 10^{25} \mathrm{~m}^{-3} \\
& N=25.0
\end{aligned}
$$

The results of Erman and Flory on the PDMS(B) material appear in Fig. 5. PDMS(B) was crosslinked with half as much $(0.1 \% \mathrm{w} / \mathrm{w})$ dicumyl peroxide as was PDMS(A); thus the responses in Figs. 4 and 5 differ; the difference is primarily in the moduli of the curves. In the range of responses shown in Figs. 4 and 5 , the differences in the network structures formed by the PDMS networks include an approximately twofold increase in stiffness and optical anisotropy for material PDMS(A) over PDMS(B), corresponding to a doubling of the amount of crosslinking agent in (A) over (B). The model simulations in Fig. 5 used the following material properties of PDMS(B):

$$
\begin{aligned}
& \eta_{0}=1.4074 \\
& \alpha=5.4 \times 10^{-31} \mathrm{~m}^{3} \\
& n=2.25 \times 10^{25} \mathrm{~m}^{-3} \\
& N=50.0
\end{aligned}
$$

In characterizing the two PDMS networks we assumed the link anistropy $\alpha$ to remain constant and allowed differences in the values of $n$ and $N$ needed to describe the curves in Figs. 4 and 5. The Erman and Flory network PDMS(A) was formed with twice

${ }^{1}$ We consider that the upturn in the nominal stress vs. stretch curve of natural rubber is due to a non-Gaussian effect and that possible crystal lization effects provide a secondary influence to the nominal stress vs. lization effects provide a secondary influe
stretch curve, as is argued in Treloar (10).
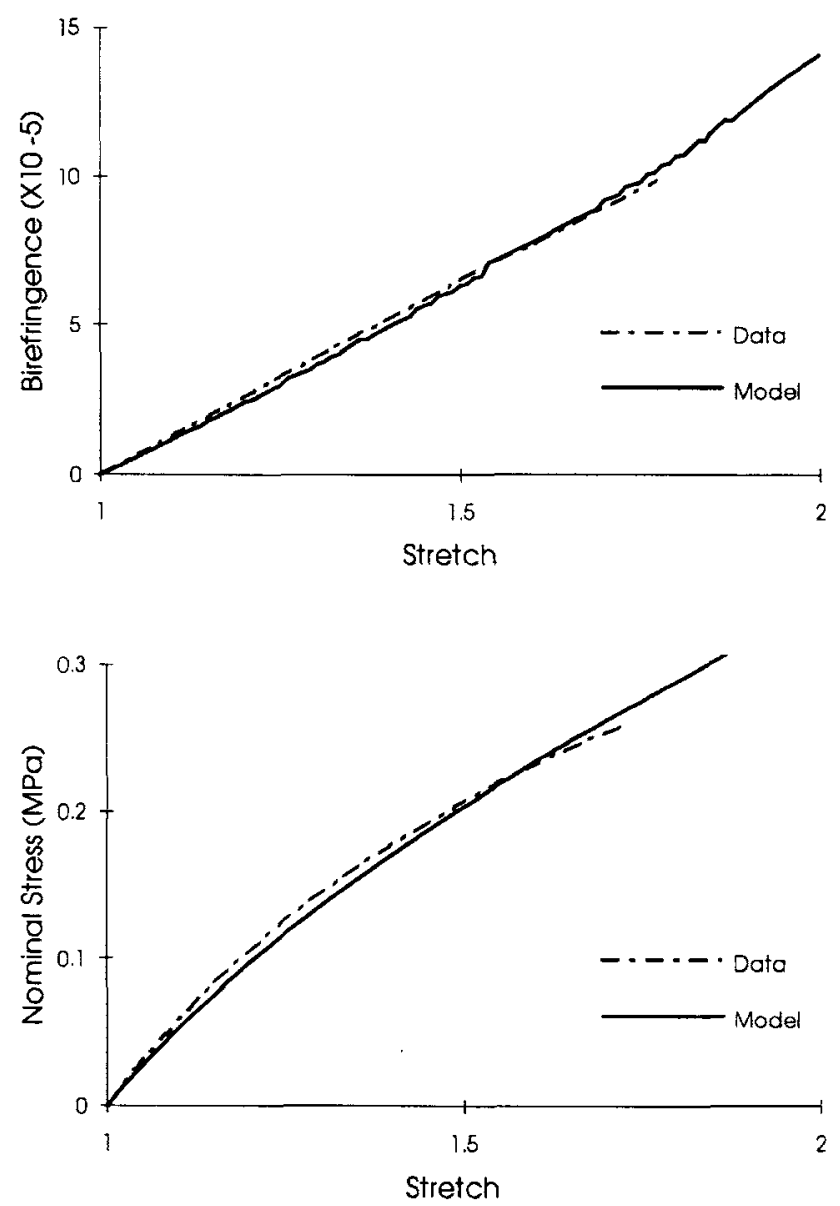

Fig. 4. Birefringence us. stretch and nominal stress vs. stretch responses of a polydimethylsiloxane network [PDMS(A) in the text] in uniaxial tension. Data from Erman and Flory (9). Simulations used Eq 14 for birefringence, and Eq 19 for nominal stress.

the amount of crosslinking agent used in their PDMS(B), which presumably resulted in twice as many crosslinks for a random crosslinking process. The values of $n$ needed in the current theory for these two PDMS networks differ by a factor of 2.0, in accordance with the increase in crosslink density of PDMS(A) over PDMS(B). Moreover, a twofold increase in the number of crosslinks must coincide with a decrease in the average length of chains between crosslinks, the extent of the decrease depends upon the nature and density of the crosslink structure. The measure of chain length provided by $N$, the number of statistical rigid links per chain, would therefore be expected to drop with the increase in crosslink density, $n$. The simulations used a twofold increase in the number of links per chain, $N$, in network PDMS(B) compared to PDMS(A) to characterize the PDMS(B) network data. The results in Figs. 4 and 5 show that the model is able to capture the birefringence vs. stretch and stress vs. stretch responses of both PDMS networks reasonably well over the entire range of extensibility reported for these networks by Erman and Flory (9). These results also show the ability of 

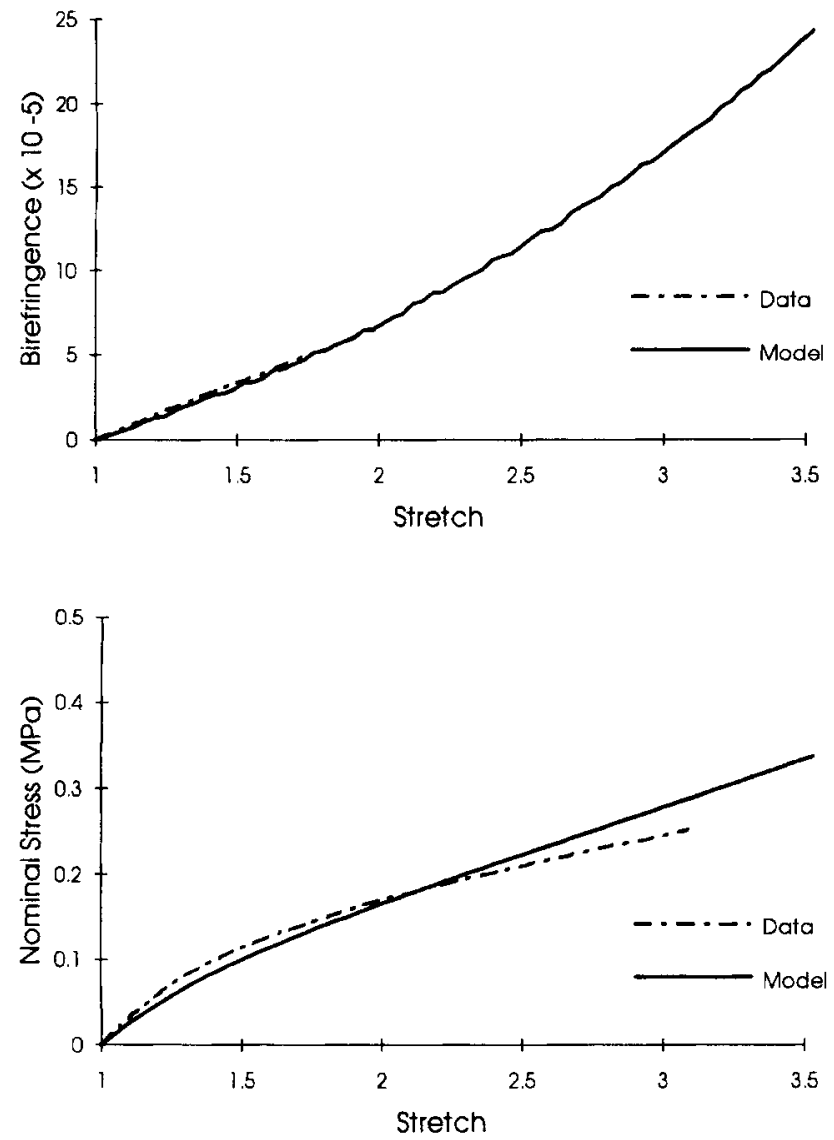

Fig. 5. Birefringence us. stretch and nominal stress vs. stretch responses of a polydimethylsiloxane network [PDMS $(B)$ in the textl in uniaxial tension. Data from Erman and Flory (9). Simulations used Eq 14 for birefringence, and Eq 19 for nominal stress.

the model to capture changes in the structural features of the network that arise from changes in the synthesis procedure. Changes in the defect structure associated with two different synthesis techniques are also to be expected; these should also affect the exact values of the properties $n$ and $N$ used to obtain the best representations of the network responses.

The data in Fig. 6 (19) show the uniaxial tension and uniaxial compression responses of a PDMS network, which is similar in response to the Erman and Flory (9) PDMS(B) data of Fig. 5. Details of the synthesis process used for this network were not reported by the authors, and thus it was assumed that the network was formed similarly to that used in Erman and Flory (9) because the uniaxial tension data show closely matched (but not exactly matched) responses. The simulations included in Fig. 6 used the material properties listed earlier for PDMS(B) to generate both the uniaxial tension and uniaxial compression nominal stress vs. stretch curves. Figure 6 demonstrates how characterization of the material constitutive response is complete with the birefringence and stress responses in uniaxial tension; the uniaxial compression response is predicted based on the model geometry and the material properties found
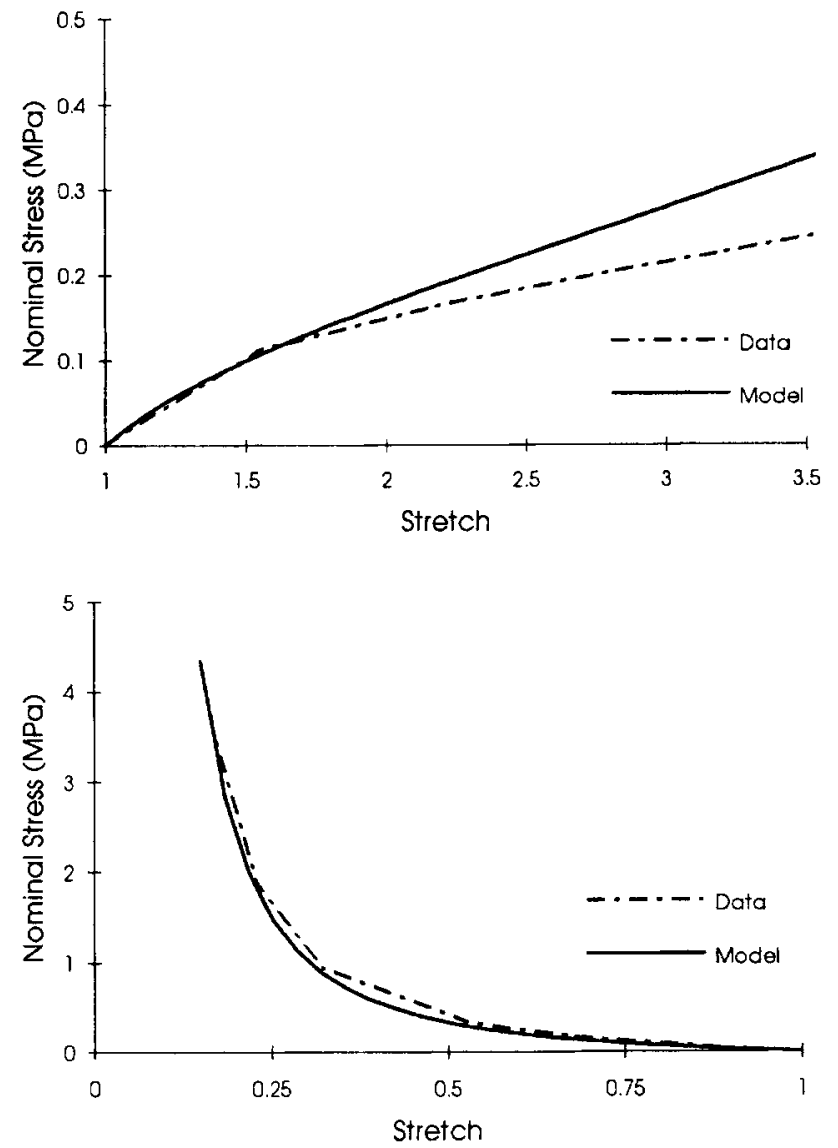

Fig. 6. Nominal stress vs. stretch responses of a polydimeth $y$ lsiloxane network [similar to PDMS $(B)$ in the text] in uniaxial tension and uniaxial compression. Data from Erman and Flory (19). Theoretical predictions use the material properties found for Fig. 5 to predict the uniaxial compression response.

from the tension test. No parametric adjustment is required in order to simulate the uniaxial compression test in Fig. 6. We are currently performing measurements of the birefringence of elastomeric networks during deformation in uniaxial compression and pure shear as well as uniaxial and biaxial tension in order that we may further investigate the use of $\mathrm{Eq} 19$ together with birefringence data to predict the current anisotropic stress response of elastomers under large deformations.

\section{CONCLUSIONS}

In this paper we have developed an internal variable-based constitutive model for the deformation response of elastomers at large strains. The model uses the experimentally measured birefringence of a deforming elastomer as a state dependent variable and the rubbery modulus of the elastomer to simulate the nominal stress response associated with the imposed deformation state. The model is shown to be successful in describing the uniaxial tension birefringence vs. stretch and stress vs. stretch responses of both natural rubber and PDMS networks. We have also provided evidence of the ability of the model to predict 
the deformation state dependent response for other deformation states using the birefringence data for the particular deformation process and the characterization parameters from the uniaxial tension test without parametric adjustments. More conclusive evidence is possible for such deformation states only when additional birefringence data in a variety of deformation states become available. We are currently generating such data for elastomeric networks via large deformation studies in uniaxial compression, plane strain compression, uniaxial extension and biaxial extension.

\section{ACKNOWLEDGMENTS}

This work is supported through a grant from the Horace H. Rackham School of Graduate Studies and the Office of the Vice President for Research at the University of Michigan, Ann Arbor, Michigan.

\section{REFERENCES}

1. M. J. Mooney, J. Appl. Phys., 11, 582 (1940)

2. P. J. Flory and J. Rehner, Jr., J. Chem Phys., 11, 512 (1943).

3. P. J. Flory and B. Erman, Macromolecules, 15, 800 (1982).

4. R. Ullman, in Advances in Elastomers and Rubber Elas ticity. J. Lal and J. E. Mark, eds., Plenum Press, New York (1986)

5. B. Erman, in Advances in Elastomers and Rubber Elas- ticity, J. Lal and J. E. Mark, eds., Plenum Press, New York (1986).

6. R. F. T. Stepto, in Advances in Elastomers and Rubber Elasticity, J. Lal and J. E. Mark, eds., Plenum Press, New York (1986).

7. E. M. Arruda and M. C. Boyce, J. Mech. Phys. Solids, 41 , 389 (1993).

8. B. Erman and P. J. Flory, Macromolecules, 16. 1601 (1983).

9. B. Erman and P. J. Flory, Macromolecules, 16, 1607 (1983).

10. L. R. G. Treloar, The Physics of Rubber Elasticity, Oxford University Press, Oxford, U.K. (1975).

11. Y. Abe, M. H. Liberman, and P. J. Flory, Macromolecules, 5. 550 (1972).

12. L. R. G. Treloar, Trans. Faraday Soc., 43, 284 (1847).

13. W. Kuhn and F. Grün, Kolloid Zeitschrift, 101, 248 (1942).

14. L. R. G. Treloar and G. Riding, Proc. Roy. Soc. London A, 369, 281 (1979).

15. K. J. Smith, Jr. and D. Puett, J. Appl Phys., 37, 346 (1966).

16. M. V. Volkenstein, Configurational Studies of Polymeric Chains, John Wiley and Sons, New York (1963).

17. D. W. Van Krevelen, Properties of Polymers: Their Corre lation With Chemical Structure, their Numerical Estimation and Prediction from Additive Group Contributions, Elsevier, New York (1990).

18. K. Nagai, J. Chem. Phys., 40, 2818 (1964).

19. B. Erman and P. J. Flory, Macromolecules, 15, 806 (1982).

Received September 1994 